\title{
A New Theory of Muscle Contraction
}

ONE of the outstanding mysteries of molecular biology is the mechanism whereby chemical energy is converted into work during muscular contraction. In spite of significant advances in the past few years in knowledge of the details of muscle structure and of the biochemical reactions involved in contraction, no comprehensive theory has yet been proposed which satisfactorily explains the molecular events involved in this process. A major difficulty lies in the complete ignorance of the nature of the basic mechanism involved. Mechanisms which have been proposed range from mechano-chemical processes, as suggested by Davies (Nature, 199, 1068; 1963), to the electrostatic mechanisms proposed by Elliott, Rome and Spencer (Nature, 226, 417 ; 1970).

Experiments on muscle contraction tend to fall into one of three distinct categories-structural, biochemical or physiological-and these apply also to contraction theories, which may explain observations from one area, but frequently give only a superficial consideration to observations from the other areas. Because results obtained in one category normally complement results obtained in the other two, a complete explanation of the contraction process can only come when results from all areas are melded into a single theory. Data from structural studies can provide the framework in which biochemical processes producing the energy conversion can take place. It is physiological data, however, which can test the relevance of any structural or biochemical theory to living muscle and its normal behaviour.

On page 533 of this issue of Nature, A. F. Huxley and R. M. Simmons propose a theory of contraction which combines observations from two of the three categoriesstructural and physiological. Their theory has evolved from A. F. Huxley's theory (Progr. Biophys., 7, 255 ; 1957) which has been reworked to take account of recent structural and physiological observations.

Huxley's original theory followed the discovery in 1954 of the sliding filament mechanism for muscle contraction. Huxley suggested that contraction resulted from the formation of actin-myosin links with specific elastic properties between the two types of filaments. The rates of making and breaking the links governed the extent and speed of the muscle's contraction. Calculations based on this theory could account for several known physiological relationships in muscle. Details of the structural components and of the biochemical reactions could not, however, be specified.

Recent experiments by Huxley and Simmons on transient tension effects in single fibres of frog muscle after quick stretches or releases have enabled them to specify certain properties of the actin-myosin links. Although Huxley and Simmons state that their general proposals can be applied to several structural models and are similar to a much earlier theory of visco-elastic behaviour of high molecular weight polymers proposed by Eyring (J. Chem Phys., 4, 283; 1936), they have based their theory on H. E. Huxley's recent structural model (Science, 164, 1356; 1969).

In H. E. Huxley's model, myosin and actin filaments slide past one another (and thus cause shortening) through the formation of "cross-bridges" between the two types of filaments. Each cross-bridge results from the attachment of a projecting part of the thick myosin filament (myosin "heads", equivalent to heavy meromyosin subfragment one) to specific sites on the thinner actin filaments. Shortening at each site is produced by a change in the angle that the cross-bridge makes with the filament axis so that the actin filament is pulled relative to the myosin filament. Individual cross-bridges are formed cyclically to produce a smooth shortening motion. The head of the myosin molecule is able to bridge the variable gap between the filaments by swinging out from a hinged region part way along the straight and otherwise rigid shaft of the molecule. A cross-bridge is formed when the myosin head attaches to an actin filament. The part of the myosin molecule which hinges outward corresponds to heavy meromyosin subfragment two.

The experiments of A. F. Huxley and Simmons suggest the existence of two elastic elements in series which they identify with the cross-bridge structure. A stiff, undamped elasticity is assigned to the straight, hinged part of the myosin molecule (subfragment two), and a damped elasticity, with a time constant of a millisecond or less, to the myosin head-actin complex. During contraction. each myosin head-actin attachment shifts through a series of stable positions (probably three), each with a lower potential energy than the last. The motion so produced, when coupled with the elasticity of the straight connecting link, can give a smoothly-changing force between the filaments. The authors have observed in experiments that a rapid change of length in the muscle fibre is associated first with a rapid tension change which parallels the length change (an undamped elasticity) and then with a (damped) recovery to a tension intermediate to the initial tension and that at the end of the length change. The tension at the end of a length change, that after the rapid recovery, and the rate of tension recovery were measured as a function of the length change imposed. The curves obtained were fitted closely by curves calculated from the theory.

To this extent, then, the theory agrees well with experimental observation. Huxley and Simmons imply, but do not demonstrate here, that their theory can also account for such established physiological data as force-velocity curves and heat production. This aspect will doubtless be covered in a fuller treatment promised for the future. The theory should also be checked quantitatively against such other observations as the length transients appearing after rapid tension changes measured by Podolsky and his colleagues (see Proc. US Nat. Acad. Sci., 64, 504 ; 1969). Equally important, is the theory compatible with the biochemical knowledge of muscle contraction-the energy requirements during contraction, and the roles of adenosine triphosphate, phosphocreatine and calcium ?

The success of any theory ultimately resides in the future experiments it stimulates. If the Huxley and Simmons theory enables physiologists and others to develop meaningful experiments to test its predictions against existing structural and biochemical knowledge, it will have done valuable service to muscle research, whatever its fate in the archives of contraction theories. 